\title{
TOWARDS NUMERICAL SIMULATION OF SNOW SHOWERS IN JET- ENGINE FUEL SYSTEMS
}

\author{
Ewen MARECHAL ${ }^{1}$, Sofiane KHELLADI, Florent RAVELET, Farid BAKIR \\ DynFluid Laboratory \\ Ecole Nationale Supérieure des Arts et Métiers \\ 151, Boulevard de l'Hôpital, 75013 Paris, FRANCE \\ ewen.marechal@ensam.eu sofiane.khelladi@ensam.eu, florent.ravelet@ensam.eu, farid.bakir@ensam.eu
}

\author{
Olivier DELEPIERRE-MASSUE \\ SNECMA \\ Rond-point René Ravaud, 77550, Réau, FRANCE \\ olivier.delepierre-massue@snecma.fr
}

\section{KEY WORDS}

Finite volume, Simple algorithm, Moving Least Squares, Particle, Two-way coupling

\begin{abstract}
Aircraft fuel systems are subject to icing at low temperatures. If the flow rate is increased, sudden releases of large quantities of ice may occur, called "snow showers". They threaten the safety of flights and have been the subject of several investigations over past years. Jet engines fuel system components may sensitive to clogging. When a snow shower happens, ice particles settle in seconds, forming a porous layer. Modelling such events involves transient hydraulics and solid dynamics. We propose to investigate numerically the dynamics of transient particle clogging. Equations of motion for the incompressible fluid phase are discretized in a high-order finite-volume context and solved using a pressure-based algorithm. The discrete phase is modelled in a Lagrangian frame. Contacts between solids are handled by a dedicated algorithm. Solid volume fraction is calculated in regions occupied by particles. Finally, two-way coupling is achieved by source terms for momentum exchange, viscous and inertial loss. $2 D$ simulation of the clogging of an ideal filter is performed.
\end{abstract}

\section{INTRODUCTION}

Ice in flight can be disastrous. A thin layer on the airfoil degrades its ability to lift and increases its drag, leading the airplane to stall even at high speeds. Ice collected on wing can also broke off and be sucked into the engines, damaging the fan blades. An airplane can roll or pitch uncontrollably if icing occurs on the horizontal or vertical stabilizer. Obstruction of pitot tube will cause the airspeed indicator to give wrong information. These events are related to weather (moisture, snow, freezing rain, and drizzle) and caused hundreds of casualties over decades.

However, fuels used in jet aircraft contain significant quantities of water. Investigation of the Boeing 777-236ER, G-YMMM crash at Heathrow in 2008 revealed that this water can create ice structures in aircraft fuel ducts at low temperatures and moderate flow rates. If the latter is increased, shear stress may tear off the ice resulting in high concentrations of particles in the fuel. Such sudden releases of large amount of ice are often referred as a "snow showers" [1].

\footnotetext{
${ }^{1}$ Corresponding author
} 
Jet engines have complex fuel systems, involving several hydraulic components sensitive to clogging e.g. filters, valves or heat exchanger inlet screens. When a snow shower occurs, ice particles settle in seconds to form a porous layer which is likely to occasion fuel flow restrictions. Nowadays, aviation safety authorities require that fuel systems must be designed so that any accumulation of water which may separate from the fuel will not cause engine malfunctioning [2]. Hence, understanding of such flow has become a rising matter of interest among jet engines manufacturers over last years.

In this paper, we propose a numerical approach to investigate dynamics of transient clogging of hydraulic components. Simulation strategies for particulate flows can be roughly classified in two categories. The first one is the Eulerian-Eulerian approach. The fluid phase and the dispersed phase are considered as interpenetrating continuum and updated using Mass, momentum and energy conservation laws. Additional constitutive equations may be required to describe interactions between particles. This approach is well suited for very small particles moving with the fluid, but is not adapted to packed beds involved in clogging.

Therefore we chose the second approach known as Eulerian-Lagrangian. Each particle, or group of similar particles (called parcel) is considered with its own position and velocity which are updated with the equation of motion. The effect of particles on the flow field is accounted through momentum and energy sources. Collisions between particles can be treated deterministically. Therefore it is possible to accurately describe how they stack on each other [3].

\section{MODELING SNOW SHOWER}

\subsection{A multiphysic, multiscale phenomenon}

Snow shower are tree-stage events. The first step is a slow (several hours) growth of ice crystal within the aircraft fuel pipes. Then, accumulated snow is unexpectedly released and carried by the fuel into the jetengine fuel system. At last, ice particles will choke fuel systems components in seconds.

\subsubsection{Ice accretion onto sub cooled surfaces}

Icing is influenced by several parameters, e.g. surface properties, temperature variations of fuel and fluid velocity. Laboratory observations show that accretion process involves the combination of growth of ice crystal from the water dissolved in the fuel (frost) and deposit of supercooled droplets (rime) [4, 5]. Cristal growth is driven by thermodynamics: higher molecular diffusion favours adsorption of water molecules to the growing crystal while thermal diffusion removes latent heat released by water solidification [6].

\subsubsection{Ice shedding}

The mechanical strength of an ice sample at a given temperature depends of the shape and size of the specimen, the way ice is formed, and how the load is applied. The fracture is initiated by the formation of a crack, depending on the crystallographic orientation, which then spread through the specimen. A generally fracture law has yet to be agreed upon, but correlations were proposed by several authors. Ice present weak tensile and shear strengths [7, 8]. Investigations following the 2008 accident demonstrated that release of accretion occurs when the fuel velocities is increased to the range of 0.7 to $0.8 \mathrm{~m} / \mathrm{s}$. Hence, ice shedding is likely caused by shear stress resulting from a drag increase [9].

\subsubsection{Clogging}

Components with small holes are obviously threatened by ice particles. This includes filters, strainers, injectors and heat exchanger inlets. It is usual to provide by-pass means to such components so that if they were to be obstructed, fuel will continue to flow at an acceptable rate through the rest of the system. Accumulation of a significant quantity of ice will form a thin layer. Resulting pressure drop may cause bypass valves to open. Therefore it is critical to know in which extent ice particles will follow the flow through the by-pass and propagate in the fuel system. Given a carrier fluid, the dynamic is driven by the shape, size and density of ice particles. According to recent studies, ice particles size is in the range of to 1-10 $\mu \mathrm{m}$ and may be considered almost neutrally buoyant in fuel [10]. 


\subsection{Replicating snow showers}

The European Aviation Safety Agency (EASA) issues certification specifications to ensure the tolerance of fuel systems to high concentration of ice particles. CS-E 560 states: «The fuel system must be designed so that any accumulation of likely quantities of water which may separate from the fuel will not cause Engine malfunctioning. » [2].

\subsubsection{Experimental setup}

In order to comply with international safety standards, an experimental setup was designed by the French jetengine manufacturer SNECMA. The equipment used to make such tests is described in more detail previously [11]. Briefly, pure water is introduced in cold kerosene fuel flow through a homemade injector. The system regulation ensure that a given quantity of water is injected at the desired volumetric flow rate (around $10000 \mathrm{ppm}$ ). It was observed that injected water froze almost immediately when the fuel temperature is below $-20^{\circ} \mathrm{C}$.

\subsubsection{Collected ice}

$80 \mathrm{~mL}$ of water were introduced in a $-45^{\circ} \mathrm{C}$ jet-engine fuel and ice was collected downstream by a $25 \mu \mathrm{m}$ filter. The flow decreased by about $20 \%$ though the filter appeared to be completely obstructed. Figure 1 shows the layer of ice accumulated on the filter and a close-up on ice particles.

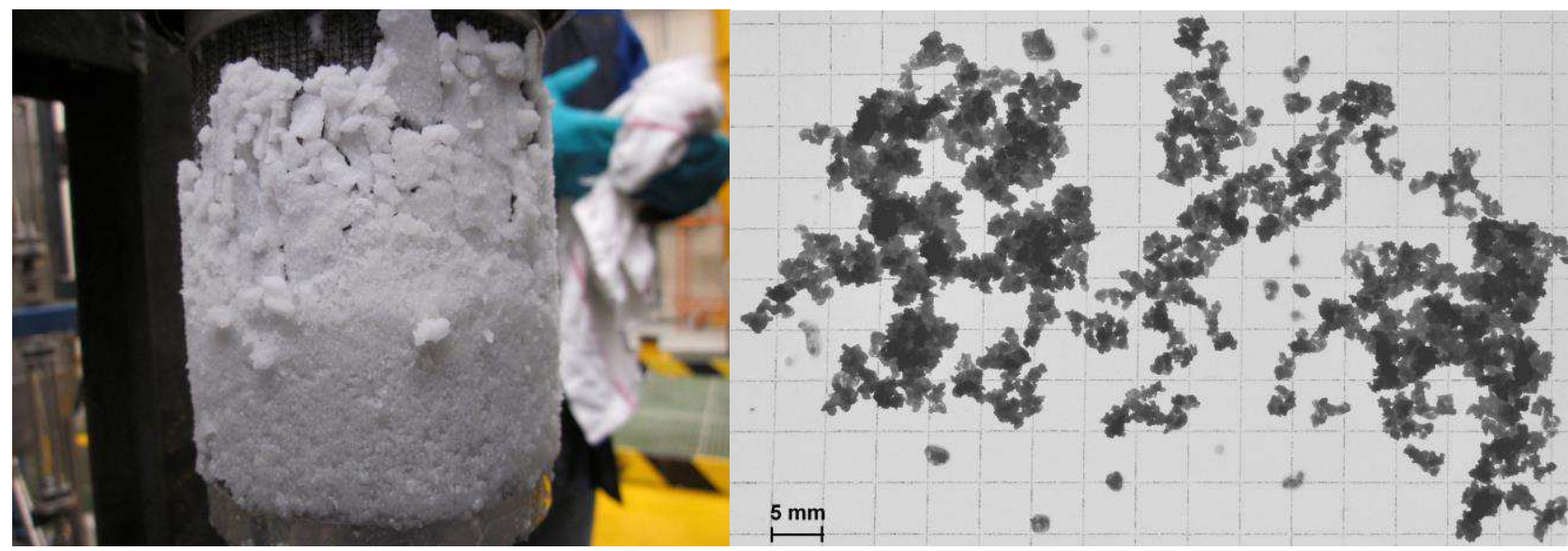

Figure 1: Left: clogging of a filter - Right: picture of particles

Ice was collected as dry as possible from the deposit and left to melt. A volume fraction up to $50 \%$ of fuel was still observed. This implies that permeability of the ice layer is a combination of compactness from particle stacking and porosity of particles themselves.

\subsection{Assumptions}

Complete modelling of a snow shower is outside of this research scope. This paper focuses on the clogging of system components by ice, which is the most critical step of the phenomenon. Furthermore, we mean to keep the model close to what have been observed in previously described tests in the perspective of future validation of the method. Hence, several assumptions are made in this study:

1. Supercooling droplets are not considered.

2. Injected water is considered as already frozen. We do not consider any freezing or melting.

3. Particles are considered already released and created at flow inlet.

4. Particle initial velocity is set equal the fuel one.

5. Particles radius is evenly distributed within the range of $150-250 \mu \mathrm{m}$.

6. Particles porosity is in the range of 0.1 to 0.5 .

7. The carrier fluid is incompressible.

8. We restrict our study to low Reynolds flow. 


\section{NUMERICAL METHOD}

\subsection{Numerical simulation of solid dynamics}

\subsubsection{Particle equation of motion}

Let consider a rigid body of mass $m$ and inertia matrix $\mathbf{J}$ submitted to total forces $\mathbf{F}$ and torques $\mathbf{T}$. Its center of mass has for coordinate and orientation $(\mathbf{X}, \mathbf{W})$. Its motion is governed by the following equations:

$$
\left\{\begin{array}{l}
m \frac{d \dot{\mathbf{X}}}{d t}=\mathbf{F} \\
\mathbf{J} \frac{d \dot{\mathbf{W}}}{d t}=\mathbf{T}
\end{array},\right.
$$

Modelling total forces $\mathbf{F}$ and torques $\mathbf{T}$ applied on a solid particle in an infinite medium has been studied extensively. Various forces are involved: drag, buoyancy, Magnus, Suffman and Basset forces, virtual mass force. In this study we only consider drag $\mathbf{F}_{\mathbf{D}}$, buoyancy $\mathbf{F}_{\mathbf{B}}$ and contact forces $\mathbf{F}_{\mathbf{C}}$. Rotation of the particle is discarded. Let $n$ denote the simulation time step of length $\Delta t$. The discretized equation of motion for a particle is:

$$
\mathbf{X}^{n+1}=\mathbf{X}^{n}+\dot{\mathbf{X}}^{n} \Delta t+\frac{1}{2} m^{-1}\left(\mathbf{F}_{\mathbf{D}}+\mathbf{F}_{\mathbf{B}}+\mathbf{F}_{\mathbf{C}}\right)^{n} \Delta t^{2},
$$

\subsubsection{Drag in a multiparticle system}

In the following, subscript $\mathrm{M}$ denotes the mixture, $\mathrm{C}$ the fluid continuum, and $\mathrm{P}$ the particle. Drag arises from the relative motion $\mathbf{V}_{R}$ between the particle and the flow:

$$
\mathbf{V}_{R}=\dot{\mathbf{X}}_{P}-\dot{\mathbf{X}}_{C}
$$

A standard relation for drag is force is:

$$
\mathbf{F}_{\mathbf{D}}=-\frac{C_{D}}{2} \rho_{C} S_{P}\left|\mathbf{V}_{R}\right| \mathbf{V}_{R},
$$

In a multiparticle system, the effect of others particles on the drag force is modelled as an increase of viscosity resulting from additional stress in the flow. We use the mixture viscosity model proposed by Clift $\& \mathrm{Al}[12]$ :

$$
\mu_{M}=\mu_{C}(1-\alpha)^{-2.5}
$$

Where $\mu$ denotes the mixture viscosity, and $\alpha$ the fraction of volume occupied by particles. Two similarity hypotheses are introduced. First, the particle Reynolds number is defined as:

$$
\operatorname{Re}_{P}=\frac{\rho_{C} d_{P}\left|\dot{\mathbf{X}}_{P}-\dot{\mathbf{X}}_{C}\right|}{\mu_{M}},
$$

Then it is assume that a similarity exists between a single-particle system and a multi-particle system. The relation for the drag coefficient is given by:

$$
C_{D}=\frac{24}{\operatorname{Re}_{P}}\left(1+\frac{\operatorname{Re}_{P}^{0.75}}{10}\right),
$$

This equation holds for particle Reynolds number lesser than 1000. Above this value, the drag coefficient $C_{D}$ should be set to a constant value of 0,44 [12]. It is also necessary to set a maximum value for $C_{D}$. Otherwise, particle velocity may exceed fluid velocity at the end of a step. Maximum drag coefficient value is related to the time step. It has been found that sedimentation speed is sensitive to this parameter.

\subsubsection{Particle interactions}

The mixture viscosity model only holds for moderate solid volume fractions. In dense flow and packed bed we need to take into account contact forces between particles. In this paper, we derive a discrete contact model from equation of motions. Let $\mathrm{i}$ and $\mathrm{j}$ denotes two particles of radius $r_{i}$ and $r_{j}$. Let $\mathbf{X}_{i}^{n}$ and $\mathbf{X}_{j}^{n}$ be their centers of mass of mass. 
We define the directional unit vector between $i$ and $j$ as:

$$
\mathbf{E}_{\mathbf{i j}}=\left(\mathbf{X}_{j}^{n}-\mathbf{X}_{i}^{n}\right) \mid \mathbf{X}_{j}^{n}-\mathbf{X}_{i}^{n} \|^{-1}
$$

Let $\mathbf{F}_{i j}=-\mathbf{F}_{j i}$ be a force exerted by particle $\mathrm{j}$ on particle i over a time step. This force is directed along $\mathbf{E}_{\mathbf{i j}}$. Writing equation of motion (2) for each particle and taking into account $\mathbf{F}_{i j}$ give the following system:

$$
\left\{\begin{array}{l}
\mathbf{X}_{i}^{n+1}=\mathbf{X}_{i}^{n}+\dot{\mathbf{X}}_{i}^{n} \Delta t+\frac{\Delta t^{2}}{2 m_{i}}\left(\mathbf{F}_{i}-\mathbf{F}_{i j}\right)=\mathbf{X}_{i}^{n}+\hat{\mathbf{V}}_{i}^{n} \Delta t+\frac{\Delta t^{2}}{2 m_{i}} \mathbf{F}_{i j} \\
\mathbf{X}_{j}^{n+1}=\mathbf{X}_{j}^{n}+\dot{\mathbf{X}}_{j}^{n} \Delta t+\frac{\Delta t^{2}}{2 m_{j}}\left(\mathbf{F}_{j}+\mathbf{F}_{i j}\right)=\mathbf{X}_{j}^{n}+\hat{\mathbf{V}}_{j}^{n} \Delta t-\frac{\Delta t^{2}}{2 m_{j}} \mathbf{F}_{i j}
\end{array},\right.
$$

Where $\hat{\mathbf{V}}_{i}^{n}$ and $\hat{\mathbf{V}}_{j}^{n}$ are called "a priori" velocity of particles $\mathrm{i}$ and $\mathrm{j}$. These velocities can be such that if no force is exerted between particles, there may be interpenetration.

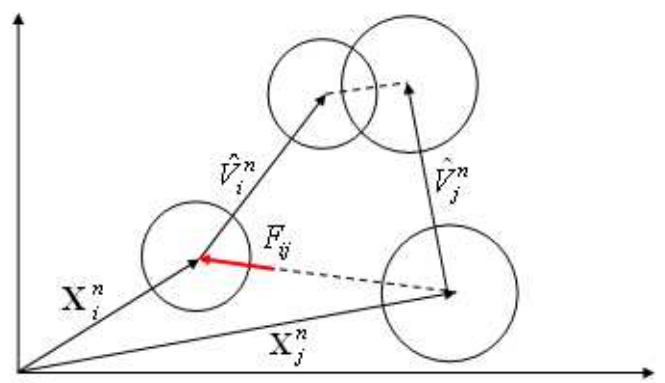

Figure 2: Particles initial position, directional vector, and a priori velocities and particles final positions given $\mathbf{F}_{i j}=0$ Subtracting equations of motion for particles $\mathrm{i}$ and $\mathrm{j}$ in (9) give the following relation:

$$
\mathbf{X}_{j}^{n+1}-\mathbf{X}_{i}^{n+1}=\left(\mathbf{X}_{j}^{n}-\mathbf{X}_{i}^{n}\right)+\left(\hat{\mathbf{V}}_{j}^{n}-\hat{\mathbf{V}}_{i}^{n}\right) \Delta t-\frac{\Delta t^{2}}{2 m_{i j}} \mathbf{F}_{i j}
$$

Where $m_{i j}=\left(m_{i}^{-1}+m_{j}^{-1}\right)^{-1}$.Now we perform the dot product of (10) with the directional vector $\mathbf{E}_{\mathbf{i j}}$ :

$$
\left(\mathbf{X}_{j}^{n+1}-\mathbf{X}_{i}^{n+1}\right) \cdot \mathbf{E}_{\mathbf{i j}}=\left(\mathbf{X}_{j}^{n}-\mathbf{X}_{i}^{n}\right) \cdot \mathbf{E}_{\mathbf{i j}}+\left(\hat{\mathbf{V}}_{j}^{n}-\hat{\mathbf{V}}_{i}^{n}\right) \cdot \mathbf{E}_{\mathbf{i j}} \Delta t-\frac{\Delta t^{2}}{2 m_{i j}}\left|\mathbf{F}_{i j}\right|,
$$

Let denote $l_{i j}^{n}$ the projected distance between particle centers of mass and $\hat{\mathbf{V}}_{i j}^{n}$ the relative a priori velocity. To guarantee that no interpenetration occurs at the end of a time step, we need to set a constraint $l_{i j}^{n+1} \geq r_{i}+r_{j}$. Introduction of this constraint in equations (11) give:

$$
l_{i j}^{n}-r_{i}-r_{j}+\hat{\mathbf{V}}_{i j}^{n} \cdot \mathbf{E}_{i j} \Delta t-\frac{\Delta t^{2}}{2 m_{i j}}\left|\mathbf{F}_{i j}\right| \geq 0,
$$

According to the velocity projection method introduced by Lefebvre in [13], finding the non-overlapping velocity field is equivalent to minimizing the following functional:

$$
J(\mathbf{V}, \lambda)=\left|\mathbf{V}-\hat{\mathbf{V}}^{n}\right|^{2}-\sum_{i<j} \lambda_{i j}\left(l_{i j}^{n}-r_{i}-r_{j}+\hat{\mathbf{V}}_{i j}^{n} \cdot \mathbf{E}_{i j} \Delta t\right),
$$

Solution of this saddle-point problem is obtained iteratively by the Uzawa algorithm:

$$
\left\{\begin{array}{c}
\mathbf{V}_{i}^{k+1}=\hat{\mathbf{V}}_{i}{ }^{n}+\sum_{i<j} \lambda_{i j}{ }^{k} \mathbf{E}_{i j} \\
\lambda_{i j}{ }^{k+1}=\max \left(0, \lambda_{i j}{ }^{k}-\frac{\omega}{\Delta t}\left(l_{i j}{ }^{n}-r_{i}-r_{j}+\hat{\mathbf{V}}_{i j}{ }^{n} \cdot \mathbf{E}_{i j} \Delta t\right)\right),
\end{array}\right.
$$

The real $\omega$ is the non-dimensional artificial time parameter. Comparison of equations (12) and (14) shows the relation between contact force and Lagrange multipliers:

$$
\lambda_{i j}=-\frac{\Delta t}{2 m_{i j}}\left|\mathbf{F}_{i j}\right|,
$$




\subsection{Numerical simulation of fluid dynamics}

Flow simulation is performed using a homemade finite volume code. The geometry is meshed so that computational cells remain greater than particles. This is essential to get cell-averaged quantities as solid volume fraction, but can lead to rather coarse meshes.

\subsubsection{Moving Least Squares approximation}

In order to have a good accuracy of the solution, we use a high order formulation based on Moving Least Squares (MLS) approximants, a technique widely used in the meshless community. Let $u(\mathbf{x})$ be a function. Its MLS approximation $\hat{u}(\mathbf{x})$ is computed from a set of $N s$ neighbouring points called stencil where the value $u_{j}$ of $u(\mathbf{x})$ is known. The number of neighbours depends of the order of accuracy and the stencil should be as compact as possible, centred around the node. The MLS approximation $\hat{u}(\mathbf{x})$ is written:

$$
\hat{u}(\mathbf{x})=\sum_{j=1}^{N s} N_{j} u_{j},
$$

High order approximate derivatives can be expressed in terms of the derivatives of the MLS shape function. The $\mathrm{n}$-th derivative of $u(\mathbf{x})$ can be expressed:

$$
\frac{\partial^{n} \hat{u}}{\partial x_{i}^{n}}=\sum_{j=1}^{N s} \frac{\partial^{n} N_{j}}{\partial x_{i}^{n}} u_{j},
$$

We refer the reader to [14] for a complete description of the computation of MLS shape functions and derivatives.

\subsubsection{Navier-Stokes equations}

Fluid motion is governed by the well-known Navier-Stokes equations. Let $\mathbf{U}=(u, v, w)^{T}$ denotes the velocity vector $\left(\mathrm{m} . \mathrm{s}^{-1}\right), \rho_{0}$ the fluid density $\left(\mathrm{kg} \cdot \mathrm{m}^{-3}\right)$, and $p$ the pressure $(\mathrm{Pa})$. In order to achieve two-way coupling between particles and fluid, we replace the fluid viscosity by previously defined mixture viscosity and introduce a source term $\mathbf{S}_{\mathbf{V}}$ to account for viscous loss within the porous layer [15]:

$$
\mathbf{S}_{\mathbf{v}}=\frac{\mu_{M}}{\varepsilon} \mathbf{U}
$$

Let $\bar{d}_{P}$ denotes the mean particle diameter and $\alpha$ the volumetric solid fraction. Parameter $\varepsilon$ is defined:

$$
\varepsilon=\frac{\bar{d}_{P}^{2}(1-\alpha)^{3}}{150 \alpha^{2}}
$$

The continuity and momentum conservation equations integrated over a control volume $\Omega$ can be written:

$$
\begin{gathered}
\oint_{\Gamma} \rho_{0} \mathbf{U} \cdot \mathbf{n} d \Gamma=0, \\
\int_{\Omega} \rho_{0} \frac{\partial \rho \mathbf{U}}{\partial t} d \Omega+\oint_{\Gamma} \mathbf{U} \cdot\left(\rho_{0} \mathbf{U} \cdot \mathbf{n}\right) d \Gamma=-\oint_{\Gamma} p \cdot \mathbf{n} d \Gamma+\oint_{\Gamma} \mu_{M}(\nabla \mathbf{U} \cdot \mathbf{n}) d \Gamma+\int_{\Omega} \mathbf{S}_{\mathbf{V}} d \Omega,
\end{gathered}
$$

Equations (20) and (21) are discretized using a novel high-order formulation based on MLS approximants introduced by Ramirez \& Al solved. The solution procedure is based on the SIMPLE algorithm [16]. We refer the interested reader to [17] for a complete description of the method.

\section{APPLICATION: CLOGGING OF A FILTER}

By way of application we consider the simple 2D case of a filter clogged by a snow shower. An ideal filter (permeable to the flow but blocking any kind of particle) is located in the middle of rectangular domain representing a segment of pipe. Domain dimensions are $2.5 \mathrm{~cm}$ (the diameter) by $10 \mathrm{~cm}$ (the length).

As inlet condition, we set the fluid velocity to $0.1 \mathrm{~m} / \mathrm{s}$ and up to 1000 particles are seed in order to have a concentration of water close to $10000 \mathrm{ppm}$. The carrier fluid has a density of $850 \mathrm{~kg} / \mathrm{m} 3$ and a viscosity of 1.10-3 Pa.s. Ice particles have a density of $917 \mathrm{~kg} / \mathrm{m} 3$ and porosity is set to 0.5 . We set the time step to $1 \mathrm{~ms}$ and perform up to 3000 iterations. 
Figure 3 shows the evolution of the velocity field $\mathbf{U}$ and solid fraction $\alpha$ over time. Particles seem to be by influenced by buoyancy, though this could be due to the maximum value imposed on drag coefficient. The deposit starts building on the bottom of the pipe, but contact forces push particles upward until equilibrium is reached. The velocity field is disturbed by particles, and a global decreased of the velocity is observed as particles settle to form a porous layer.



Figure 3: $2 \mathrm{D}$ simulation of transient clogging of a filter. Left: solid volume fraction field. Right: velocity magnitude field. Snapshots taken at time $=0.5,1.0,2.0$ and 3.0 seconds.

Figure 4 shows the network of contact forces between particles. Because diameters are randomly generated between of 150 and $250 \mu \mathrm{m}$, the stack is irregular; Therefore some particles close to the filter are under the full load of the drag cumulated by the stack of particles while other are not.

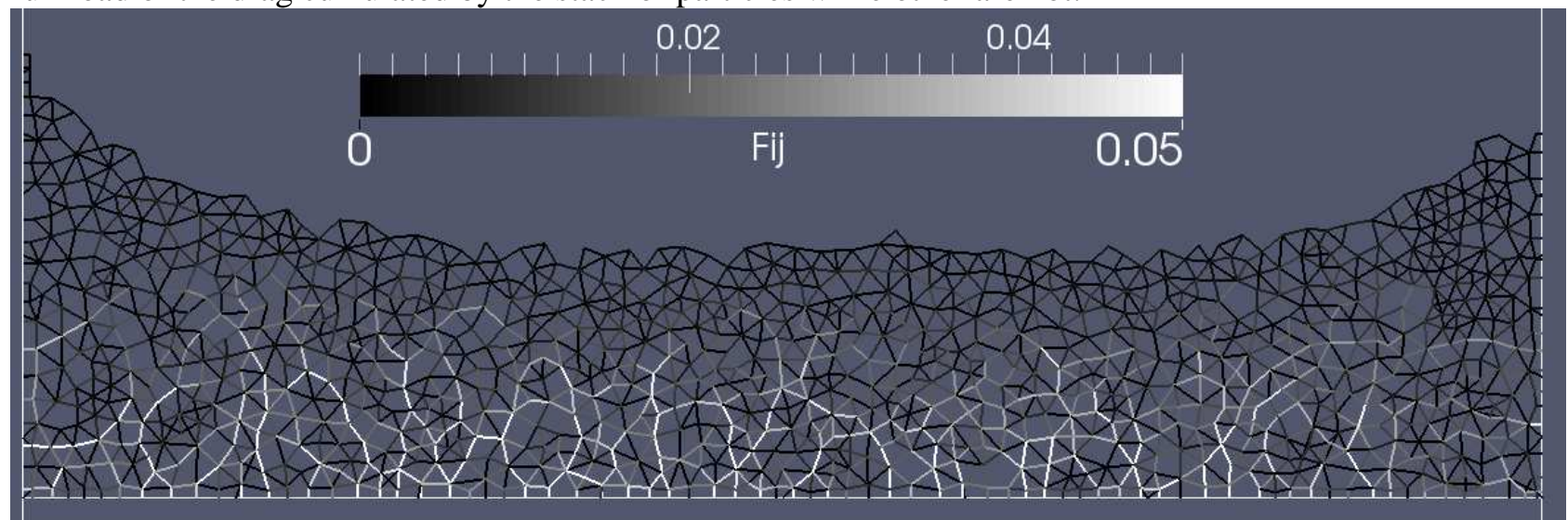

Figure 4: 2D simulation of transient clogging of a filter. Network of contact forces between particles. 


\section{CONCLUSION}

We proposed a method to simulate clogging of components by snow showers. A standard formulation is used to describe particle dynamics and retroaction of particles on the flow. Contacts forces between particles are introduced to describe the behavior of the packed bed formed by accumulating particles. A first simulation performed on the simple case of a $2 \mathrm{D}$ ideal filter in a pipe is in qualitatively consistent with experimental observations of snow shower dynamics. However, the method has to be extended in $3 \mathrm{D}$ and confronted quantitatively to available experimental data. The novel high order formulation used for computation of fluid dynamics should allow handling of large and complex geometries.

\section{ACKNOWLEDGEMENTS}

This research was carried out within the framework of a joint supervised doctoral thesis (grant \#2011/0411). The first author is grateful to SNECMA for funding and would like to thank colleagues of DynFluid laboratory for their support.

\section{REFERENCES AND CITATIONS}

[1] Sleight P.A., Carter, R.D.G., (2010) Report on the accident to Boeing 777-236ER, G-YMMM, at London Heathrow Airport on 17 January 2008. Air Accidents Investigation Branch, Department of Transport (UK).

[2] (2010) Certification Specification for Engines CS-E, Amendment 3, European Aviation Safety Agency.

[3] Multiphase flow.

[4] Baena, S., Lawson C.P., Lam J.K.-W., (2012) Cold fuel test rig to investigate ice accretion on different pump inlet filter-mesh screens, 28th International Congress of the Aeronautical Sciences, ICAS paper.

[5] Murray, B.J., Broadley, S.L., Morris, G.J. (2011) Supercooling of water droplets in jet aviation fuel. Fuel.

[6] Hobbs, P.V., (2010) Ice Physics, Oxford Classic Texts in the Physical Sciences.

[7] Butkovich, T.R., (1945) The ultimate strenght of ice. USA Snow Ice and Permafrost Research Establishment Res. Rep 9,12.

[8] Jellinek, H.H.G., (1958) The influence of imperfections on the strength of ice. Proc. Phys. Soc. 71.

[9] Reid, M., (2013) Engine fuel system tolerance to fuel born ice. Managing water and ice in aviation fuel under low temperature conditions, Seminar proceedings.

[10] Lam, J.K-W., (2013) WAFCOLT - Water behavior in aviation fuel under low temperature conditions. EASA Reasearch Project 2010/01.

[11] Marechal, E., Perrin, J.Y., (2014) Dispositif de simulation d'une introduction de paquets de glace dans un moteur.

[12] Clift, R., Grace, J.R., Weber, M.E., (1978) Bubbles, Drops and Particles. Academic Press.

[13] Lefebvre A., Lefebvre-Lepot, A., (2007) Modélisation numérique d'écoulements fluide/particles.

[14] Khelladi S., Nogueira X., Bakir F., Colominas I., (2011) Toward a Higher-Order Unsteady Finite Volume Solver Based on Reproducing Kernel Particle Method. Computer Methods in Applied Mechanics and Engineering, 200:2348-2362.

[15] Ergun, S., (1952) Fluid flow through packed columns. Chem. Eng. Prog. 48(2)89-94.

[16] Patankar, S.V., Spalding, D.B., A Calculation Procedure for Heat, Mass and Momentum Transfer in Three Dimensional Parabolic Flows. International Journal Heat Mass Transfer 15:1787-1806 (1972)

[17] Ramirez L., Nogueira, X., Khelladi, S., Chassaing, J-C., Colominas, I., A new higher-order finite volume method based on Moving Least Squares for the resolution of the incompressible Navier-Stokes equations on unstructured grids. 